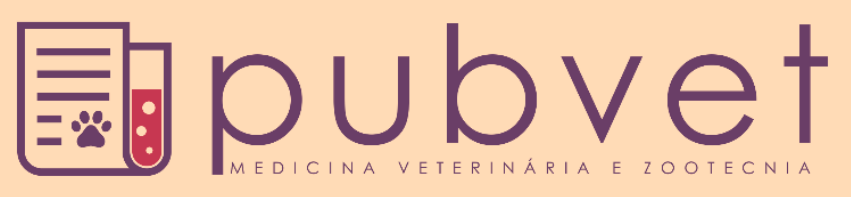

https://doi.org/10.31533/pubvet.v15n05a815.1-7

\title{
Análise de aspectos da saúde de discentes em duas escolas municipais do médio sertão alagoano
}

\author{
Joseane Barbosa Clemente Gama ${ }^{1}$, João Paulo Vieira Machado ${ }^{2 *} \bullet \mathbb{D}$, Pedro Dantas Lima ${ }^{2} \bullet \mathbb{D}$, \\ Gloria Isabel Lisboa da Silva ${ }^{2} \bullet$ (D), Israel Gomes de Amorim Santos ${ }^{3} \bullet$ (D)
}

${ }^{1}$ Graduanda do curso de Ciências Biológicas, Universidade Estadual de Alagoas - UNEAL, Campus III, Palmeira dos Índios - AL Brasil; ${ }^{2}$ Graduandos do curso de Ciências Biológicas, Universidade Estadual de Alagoas - UNEAL, Campus II, Santana do Ipanema - AL Brasil; ${ }^{3}$ Professor Adjunto do curso de Ciências Biológicas, Universidade Estadual de Alagoas - UNEAL, Campus II, Santana do Ipanema - AL Brasil. *Autor para correspondência, E-mail: joao.p.v.machado@gmail.com

Resumo. A escola é uma instituição de grande influência na formação de um indivíduo, ela também acompanha as necessidades da situação de saúde através de programas voltados para orientações e análises do bem-estar dos alunos. O objetivo deste estudo foi analisar aspectos da saúde de alunos de duas turmas de $9^{\circ}$ ano localizadas em dois municípios do médio sertão alagoano. Trata-se de um estudo epidemiológico descritivo de cunho transversal realizado em duas escolas públicas, tendo como instrumento de coleta de dados um questionário utilizado anteriormente na Pesquisa Nacional de Saúde do Escolar (PeNSE). Participaram deste estudo 71 estudantes, totalizando $62 \%$ do sexo feminino, $36,6 \%$ do sexo masculino e $1,4 \%$ que não se identificou. Com relação à alimentação foi observado que a maioria dos alunos consome feijão todos os dias e 36,6\% não consomem a merenda escolar. Sobre o uso de cigarro, álcool e drogas ilícitas, foi constatado que a maioria $73,24 \%$ já experimentou alguma bebida alcoólica e $9,96 \%$ já fumaram. Sobre sua saúde sexual e reprodutiva apenas $26,8 \%$ responderam já ter tido relações sexuais. Desta forma se faz necessário as ações de saúde nas escolas estudadas principalmente relacionadas ao consumo de bebidas alcoólicas, orientação sexual e alimentação saudável, pois a instrução e a conscientização têm um papel extremamente importante para a disseminação de hábitos saudáveis.

Palavras-chave: Educação em saúde, PeNSE, qualidade de vida

\section{Analysis of health aspects of students in two municipal schools in the Region Médio Sertão Alagoano}

Abstract. The school is an institution of great influence in the formation of an individual, it also monitors the needs of the health situation through programs aimed at orienting and analyzing the well-being of students. The aim of this study was to analyze health aspects of students from two 9th grade classes located in two municipalities in the middle of the Alagoas backlands. This is a cross-sectional descriptive epidemiological study carried out in two public schools, using a questionnaire used previously in the Pesquisa Nacional de Saúde do Escolar (PeNSE). 71 students participated in this study, totaling 62\% female, $36.6 \%$ male and $1.4 \%$ who did not identify themselves. Regarding food, it was observed that most students consume beans every day and 36.6\% do not consume school meals. Regarding the use of cigarettes, alcohol and illicit drugs, it was found that the majority $73.24 \%$ had already tried some alcoholic drink and $9.96 \%$ had already smoked. Regarding their sexual and reproductive health, only $26.8 \%$ answered that they had already had sexual intercourse. Thus, it is necessary to take health actions in the schools studied, mainly related to the consumption of alcoholic beverages, sexual orientation and healthy eating, as 
education and awareness play an extremely important role in the dissemination of healthy habits.

Keywords: Health education, PeNSE, quality of life

\section{Análisis de aspectos de la salud de alumnos de dos colegios municipales de la región Médio Sertão Alagoano}

Resumen. La escuela es una institución de gran influencia en la formación de un individuo, también monitorea las necesidades de la situación de salud a través de programas dirigidos a orientar y analizar el bienestar de los estudiantes. El objetivo de este estudio fue analizar los aspectos de salud de los estudiantes de dos clases de $9^{\circ}$ grado ubicadas en dos municipios en la región del Medio Sertão Alagoano. Se trata de un estudio epidemiológico descriptivo transversal realizado en dos escuelas públicas, manejando un cuestionario utilizado previamente en la Pesquisa Nacional de Saúde do Escolar (PeNSE). En este estudio participaron 71 estudiantes, totalizando $62 \%$ mujeres, $36,6 \%$ hombres y $1,4 \%$ que no se identificaron. En cuanto a la alimentación, se observó que la mayoría de los estudiantes consume frijoles todos los días y el 36,6\% no consume las comidas escolares. En cuanto al uso de cigarrillos, alcohol y drogas ilícitas, se constató que el 73,24\% ya había probado alguna bebida alcohólica y el $9,96 \%$ ya había fumado. En cuanto a su salud sexual y reproductiva, solo el $26,8 \%$ respondió que ya había tenido relaciones sexuales. Por ello, es necesario realizar acciones de salud en las escuelas estudiadas, principalmente relacionadas con el consumo de bebidas alcohólicas, la orientación sexual y la alimentación saludable, ya que la educación y la sensibilización juegan un papel de suma importancia en la difusión de hábitos saludable.

Palabras clave: Educación para la salud, PeNSE, calidad de vida

\section{Introdução}

A saúde não consiste apenas na ausência de doença ou enfermidade, mas sim em um estado de completo bem-estar físico e mental (OMS, 1948). Sendo assim, os hábitos e aprendizagens adquiridos pelas pessoas repercutem sobre o seu comportamento durante a vida. Isso começa a ser formado pelo convívio em família, mas sofre grandes influências quando estes passam a frequentar a escola e conviverem em sociedade.

O período entre o final da infância e adolescência, compreendido entre os anos finais do ensino fundamental e ensino médio, é um período de enormes mudanças, descobertas, novas experiências e criação de hábitos para os jovens. Entretanto, muitas dessas novidades podem influenciar negativamente na saúde, tais como o consumo de alimentos inadequados, uso de cigarro e álcool entre outros (Oliveira et al., 2017).

Segundo dados da Pesquisa Nacional de Saúde do Escolar (Brasil, 2015), 18,4\% dos estudantes entrevistados responderam positivamente quanto ao uso de cigarros, $55,5 \%$ ao de bebidas alcoólicas e $27,5 \%$ em relação a primeira relação sexual. Nesse contexto, a escola como espaço de construção e formação do pensamento crítico, de valores pessoais, crenças e do conhecimento de mundo, tem papel fundamental na saúde dos estudantes. Desta forma, o Programa Saúde da Escola (PSE) é uma importante ferramenta para as ações a serem desenvolvidas nas escolas, que busquem o desenvolvimento de práticas saudáveis e o bem-estar dos educandos (Brasil, 2011).

Tendo em vista que uma escola promotora de saúde é aquela que fortalece constantemente a sua capacidade como ambiente saudável para viver, aprender e trabalhar (WHO, 2020), o objetivo deste estudo foi analisar aspectos da saúde de alunos das turmas de $9^{\circ}$ ano de duas escolas públicas localizadas em dois municípios do médio sertão de Alagoas, Brasil. 


\section{Material e métodos}

Trata-se de um estudo epidemiológico descritivo de cunho transversal realizado em duas escolas públicas, localizadas nos municípios de Dois Riachos e Senador Rui Palmeira, ambas pertencentes ao médio sertão do estado de Alagoas.

Dois Riachos possui uma população de 10.880 habitantes e uma área de $141.69 \mathrm{~km}^{2}$, o IDH é de 0,532. Na área educacional, o município dispõe de 31 escolas, 25 com ensino de educação infantil com 378 alunos e 30 de ensino fundamental com 2.495 alunos matriculados. (IBGE, 2010). Senador Rui Palmeira tem 13.047 habitantes, com área territorial de $341.989 \mathrm{~km}^{2}$, o IDH do município é de 0,518. Quanto à educação, em Senador Rui Palmeira, segundo dados do IBGE (2015), existem apenas escolas públicas, com 349 matrículas do ensino pré-escolar, 2.869 do ensino fundamental, 504 no médio (IBGE, $\underline{2010})$.

Para a escolha das escolas alvo foram utilizados como critérios de inclusão, a adesão das escolas a projetos e pesquisas externas e pertencerem à rede pública de ensino do município. A partir destes critérios, foram selecionadas para participação no estudo uma escola da zona urbana do município de Dois Riachos e uma da zona rural do município de Senador Rui Palmeira.

O instrumento de coleta de dados utilizado foi um questionário adaptado da Pesquisa Nacional de Saúde do Escolar (PeNSE). Esse questionário é aplicado pelo IBGE. Os participantes não são obrigados a responder o questionário e os que o fazem têm um tempo máximo de 40 minutos. O questionário do PeNSE é composto por variáveis relativas a idade, gênero, escolaridade dos pais, saúde sexual e reprodutiva, saúde bucal, o consumo alimentar, imagem corporal, o uso de cigarro, de bebidas alcoólica e drogas, atividade física, saúde mental e uso de serviços de saúde. Foram utilizadas neste estudo as variáveis idade, gênero, saúde sexual e reprodutiva, o consumo alimentar, imagem corporal, o uso de cigarro, de bebidas alcoólica e drogas. O questionário foi aplicado para alunos do $9^{\circ}$ ano e para fins do anonimato das escolas participantes foram utilizados os codinomes "Escola A" e "Escola B".

\section{Resultados e discussão}

Aceitaram participar da pesquisa e responderam ao questionário 71 alunos, sendo 26 pertencentes à Escola A e 45 a Escola B. Deste total 62\% (44) eram do gênero feminino, 36,6\% (26) masculino e 1,4\% não informou o gênero. Com relação a idade, a maioria $60,5 \%$ (43) dos participantes pertencia a faixa etária de 13 a 15 anos, 29,6\% (21) de 16 a 18 anos e 9,9\% (7) tinha 19 anos ou mais (Tabela 1).

Tabela 1. Características sociodemográficas dos participantes do estudo

\begin{tabular}{lcccccc}
\hline & \multicolumn{2}{c}{ Escola A } & \multicolumn{2}{c}{ Escola B } & Total \\
Variáveis & $\mathrm{N}$ & $\%$ & $\mathrm{~N}$ & $\%$ & $\mathrm{~N}$ & $\%$ \\
\hline Gênero & & & & & & 36,6 \\
Masculino & 9 & 34,6 & 17 & 37,8 & 26 & 62 \\
Feminino & 16 & 61,5 & 28 & 62,2 & 44 & 1,4 \\
Não Informado & 1 & 3,8 & 0 & 0 & & 60,5 \\
\hline Faixa Etária, anos & & & & & 43 & 29,6 \\
13 a 15 & 8 & 30,8 & 35 & 20 & 21 & 9,9 \\
16 a 18 & 12 & 46,2 & 9 & 2 & 7 & \\
19 ou mais & 6 & 23,1 & 1 & & & \\
\hline
\end{tabular}

A idade dos participantes deste estudo, faixa etária entre 13 e 15 anos, não diferiu da idade dos alunos que participaram do PeNSE, que mostrou que a maioria dos alunos do $9^{\circ}$ ano pertenciam a esta faixa etária (Brasil, 2015). Desta forma, a maioria dos discentes participantes do estudo estavam dentro de um fluxo regular idade-série para este ano de escolarização na educação básica, embora seja preocupante o número de estudantes com idade entre 16 a mais de 19 anos que ainda estavam matriculados nessa série na época do estudo.

Quando questionados quanto à frequência semanal de consumo de feijão (Tabela 2), 74,6\% (53) dos entrevistados responderam consumir a semente todos os dias da semana. Esse quantitativo difere dos resultados encontrados no PeNSE, onde cerca de $60,7 \%$ dos escolares pesquisados em todas as regiões do país consumiam o feijão em todos os dias da semana (Brasil, 2015). Entretanto, em escolares do Rio 
Grande do Sul, evidenciou-se que quanto aos padrões alimentares 77,3\% dos participantes apresentaram consumo frequente de feijão, valor elevado assim como nesta pesquisa (Correa et al., 2017). Essa periodicidade no consumo do feijão é muito importante, sobretudo, quando se considera o poder nutricional desta semente e a sua disponibilidade no país. Atrelado a isso, o perfil nutricional, escolhas e seleção de alimentos, estão ligados, principalmente, às características culturais das diversas regiões do país (Pinheiro et al., 2004).

Com relação ao consumo da merenda escolar (Tabela 2), 36,6\% (26 alunos) afirmaram não consumirem a comida da escola, enquanto os que o faz todos os dias representaram 18,3\% (13 alunos) dos entrevistados. É imperativo que o tema alimentação seja trabalhado nestas escolas, visando a disseminação dos bons hábitos alimentares e consequentemente propiciando maior interesse desses discentes pela merenda escolar que é especialmente elaborada por nutricionista, uma vez que ela deve ser preparada com alimentos saudáveis e nutritivos, sendo que seu consumo pode diminuir o consumo de alimentos danosos para a saúde (Souza et al., 2011).

Tabela 2. Consumo e comportamento alimentar dos estudantes participantes do estudo

\begin{tabular}{|c|c|c|c|c|c|c|}
\hline \multirow[b]{2}{*}{ Variáveis } & \multicolumn{2}{|c|}{ Escola A } & \multicolumn{2}{|c|}{ Escola B } & \multicolumn{2}{|c|}{ Total } \\
\hline & $\mathrm{N}$ & $\%$ & $\mathrm{~N}$ & $\%$ & $\mathrm{~N}$ & $\%$ \\
\hline \multicolumn{7}{|c|}{ Consumo de feijão durante a semana } \\
\hline 1 a 3 dia & 2 & 7,7 & 3 & 6,7 & 5 & 7,0 \\
\hline 4 a 6 dias & 3 & 11,5 & 6 & 13,3 & 9 & 12,7 \\
\hline Todos os dias & 20 & 76,9 & 33 & 73,3 & 53 & 74,6 \\
\hline Não comi & 1 & 3,8 & 3 & 6,7 & 4 & 5,6 \\
\hline \multicolumn{7}{|c|}{ Consumo de legumes ou verduras } \\
\hline 1 a 3 dia & 12 & 46,2 & 18 & 40,0 & 30 & 42,3 \\
\hline 4 a 6 dias & 4 & 15,4 & 7 & 15,6 & 11 & 15,5 \\
\hline Todos os dias & 4 & 15,4 & 13 & 28,9 & 17 & 23,9 \\
\hline Não comi & 6 & 23,1 & 7 & 15,6 & 13 & 18,3 \\
\hline \multicolumn{7}{|c|}{ Consumo da merenda escolar } \\
\hline Todos os dias & 9 & 34,6 & 4 & 8,9 & 13 & 18,3 \\
\hline 1 a 2 dias & 1 & 3,8 & 8 & 17,8 & 9 & 12,7 \\
\hline 3 a 4 dias & 2 & 7,7 & 5 & 11,1 & 7 & 9,9 \\
\hline Não costumo comer & 10 & 38,5 & 16 & 35,6 & 26 & 36,6 \\
\hline Raramente & 3 & 11,5 & 12 & 26,7 & 15 & 21,1 \\
\hline Não Respondeu & 1 & 3,8 & 0 & 0 & 1 & 1,4 \\
\hline
\end{tabular}

Em relação a atividade sexual (Tabela 3), 26,8\% (19) dos entrevistados já haviam iniciado sua vida sexual, dado preocupante, tendo em vista a faixa etária da maioria (Tabela 1). A iniciação sexual precoce em adolescentes escolares também foi relatada por outros autores (Castro et al., 2017; Silva et al., 2015a; Silva et al., 2015b). Em um estudo realizado em Alagoas, menos de 15\% das meninas afirmaram ter tido relação sexual, enquanto os meninos, mais de 30\%, afirmaram o mesmo (Brasil, 2015). As diferenças no início da vida sexual de homens e mulheres pode ser mais bem compreendida por questões de gênero do que por questões biológicas do sexo. Isso deve-se ao fato de que o sexo é apresentado desde muito cedo para os homens como algo apropriado a se fazer, enquanto que para as mulheres como algo inapropriado, portanto eles recebem fortes estímulos para a iniciação sexual precoce do que as mulheres (Silva et al., 2015a).

A respeito da orientação sobre prevenção de gravidez e Infecções Sexualmente Transmissíveis (IST's) (Tabela 3), de todos os entrevistados, 63,4\% (45) e 71,8\% (51), respectivamente, afirmaram ter recebido aconselhamento na escola sobre o assunto. Isso é questão importante para análise, pois o número está um pouco abaixo da pesquisa nacional que demonstrou uma média entre 70 a $80 \%$ dos entrevistados (Brasil, 2015). Além disso, alguns autores apontaram em seus estudos que a maioria dos estudantes têm pouco conhecimento sobre o assunto ou dúvidas em relação às formas de transmissão e prevenção (Brêtas et al., 2009; Silva et al., 2015c; Silva et al., 2016).

Quando perguntados sobre o uso de drogas ilícitas, todos afirmaram nunca ter experimentado qualquer uma destas drogas. Já em relação ao uso de cigarro 9,96\% (7) dos entrevistados responderam já ter usado. No entanto, o mais preocupante é o consumo de bebida alcoólica, pois $73,24 \%$ (52) 
afirmaram ter consumido algum tipo de bebida alcoólica. Em alguns estados do país, diferentes autores relatam a prevalência do uso de álcool e tabaco entre escolares (Cardoso \& Malbergier, 2014; Elicker et al., 2015; Paz et al., 2018). Uma pesquisa com dados do PeNSE apresentou que 71,4\% dos estudantes do nono ano já haviam experimentado bebida alcoólica, dados um pouco abaixo dos encontrados pelo presente estudo (Malta et al., 2011). Este quadro evidencia que a bebida alcoólica é apresentada ao jovem muito precocemente, o que pode gerar problemas futuros, tanto para a saúde dos indivíduos como para o comportamento em sociedade.

Tabela 3. Comportamento sexual dos estudantes do $9^{\text {a }}$ ano das escolas estudadas

\begin{tabular}{lcccccc}
\hline & \multicolumn{2}{c}{ Escola A } & \multicolumn{2}{c}{ Escola B } & \multicolumn{2}{c}{ Total } \\
Variáveis & $\mathrm{N}$ & $\%$ & $\mathrm{~N}$ & $\%$ & $\mathrm{~N}$ & $\%$ \\
\hline Relação sexual alguma vez na vida & & & & & & \\
\hline Sim & 8 & 30,8 & 11 & 24,4 & 19 & 26,8 \\
Não & 18 & 69,2 & 34 & 75,6 & 52 & 73,2 \\
\hline $\begin{array}{l}\text { Orientação sobre prevenção a gravidez na } \\
\text { escola }\end{array}$ & & & & & & \\
\hline Sim & 16 & 61,5 & 29 & 64,4 & 45 & 63,4 \\
Não & 9 & 34,6 & 16 & 35,6 & 25 & 35,2 \\
Não sei & 1 & 3,8 & 0 & 0 & 1 & 1,4 \\
\hline Orientação sobre DSTs na escola & & & & & & \\
Sim & 19 & 73,1 & 32 & 71,1 & 51 & 71,8 \\
Não & 1 & 3,8 & 2 & 4,4 & 3 & 4,2 \\
Não sei & 6 & 23,1 & 11 & 24,4 & 17 & 23,9 \\
\hline
\end{tabular}

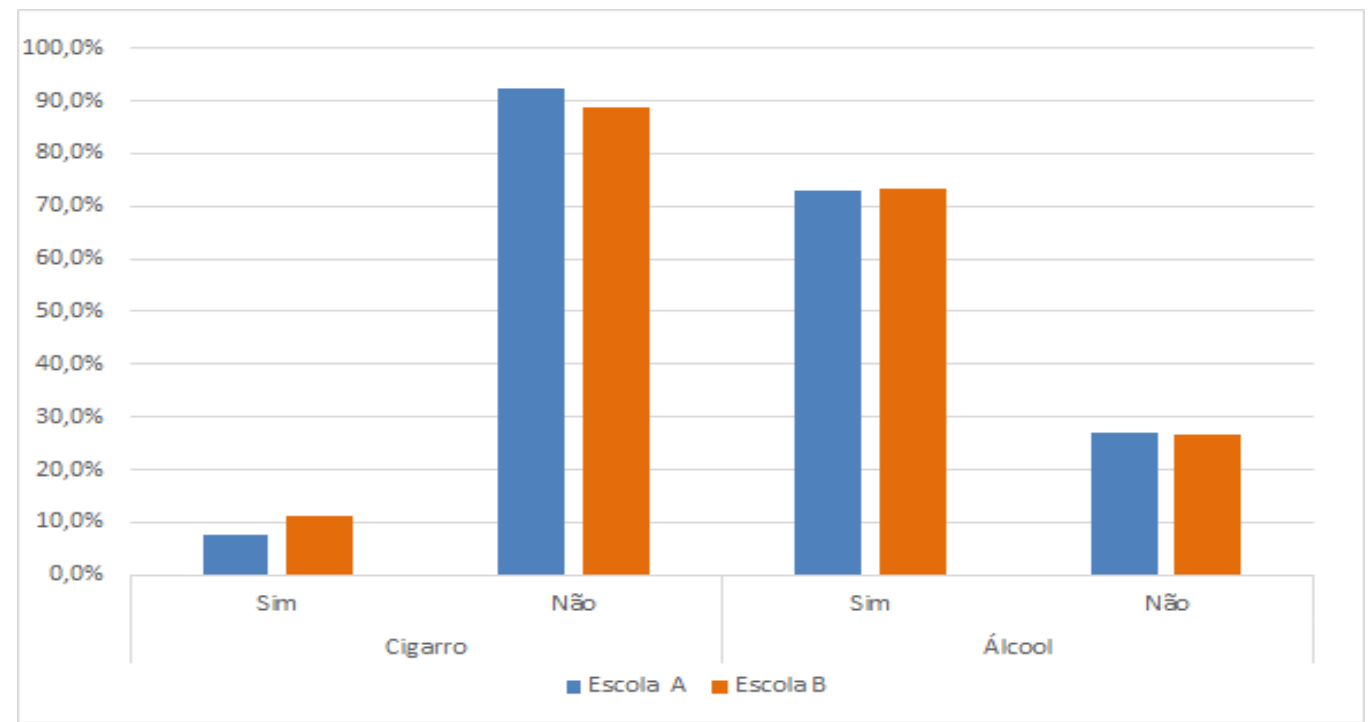

Figura 1. Uso de substâncias psicoativas por estudantes das escolas estudadas.

Por último, quando perguntados sobre a imagem corporal, 63,38\% (45) responderam considerar o corpo normal, $26,76 \%$ (19) afirmaram serem magros, 5,63\% (4) muito magros e $1,41 \%$ (1) gordo. Percebe-se então que houve maior percentual de alunos satisfeitos com a imagem corporal, por considerarem seus corpos normais, assim como descrito na pesquisa entre adolescentes escolares do ensino fundamental da rede pública estadual de Canoas/RS, onde em relação à imagem corporal, 1.091 (74,7\%) estavam satisfeitos (Lemes et al., 2018). No entanto, dois estudos, um realizado com escolares do Rio Grande do Sul e o outro estudo com universitários apresentaram respectivamente 71,5\% e 59,8\% de insatisfação dos participantes com o próprio corpo (Finato et al., 2013; $\underline{\text { Silva et al., 2019). }}$ ).

\section{Conclusões}

Os resultados encontrados apontam números preocupantes no que diz respeito ao consumo de bebida alcoólica, início precoce da vida sexual e o não consumo de merenda escolar. Esses fatores podem estar relacionados a falta ou carência de propostas de intervenção destas escolas que almejam a divulgação de temáticas relacionadas aos cuidados com a saúde. Fica evidente, portanto, que se faz necessário ações 
em saúde nas escolas estudadas, visando a conscientização sobre as práticas saudáveis, não só para o corpo estudantil, mas também para todos os envolvidos no ambiente institucional.

\section{Referências}

Brasil. (2010). IBGE. Censo demográfico. Instituto Brasileiro de Geografia e Estatística (IBGE).

Brasil. (2011). Programa Saúde na Escola: tecendo caminhos da intersetorialidade. Ministério da Saúde.

Brasil. (2015). Pesquisa Nacional de Saúde do Escolar: Avaliação do estado nutricional dos escolares do $9^{\circ}$ ano do ensino fundamental, dos municípios das capitais e Distrito Federal. Ministério do Planejamento, Orçamento e Gestão; Instituto Brasileiro de Geografia e Estatística (IBGE).

Brêtas, J. R. S., Ohara, C. V. S., Jardim, D. P., \& Muroya, R. L. (2009). Knowledge of STD/AIDS among adolescent students. Revista Da Escola de Enfermagem Da USP, 43(3), 551-557. https://doi.org/10.1590/S0080-62342009000300008.

Cardoso, L. R. D., \& Malbergier, A. (2014). Problemas escolares y el consumo de alcohol y otras drogas entre adolescentes. Psicologia Escolar e Educacional, 18(1), 27-34. https://doi.org/10.1590/S141385572014000100003.

Castro, J. F. L., Araújo, R. C., \& Pitangui, A. C. R. (2017). Sexual behavior and practices of school adolescents in Recife city, Brazil. Journal of Human Growth and Development, 27(2), 219-227. https://doi.org/10.7322/jhgd.112645.

Correa, R. S., Vencato, P. H., Rockett, F. C., \& Bosa, V. L. (2017). Dietary patterns: are there differences between children and adolescents? Ciência \& Saúde Coletiva, 22, 553-562.

Elicker, E., Palazzo, L. dos S., Aerts, D. R. G. C., Alves, G. G., \& Câmara, S. (2015). Uso de álcool, tabaco e outras drogas por adolescentes escolares de Porto Velho-RO, Brasil. Epidemiologia e Serviços de Saúde, 24, 399-410. https://doi.org/10.5123/S1679-49742015000300006.

Finato, S., Rech, R. R., Migon, P., Gavineski, I. C., Toni, V., \& Halpern, R. (2013). Insatisfação com a imagem corporal em escolares do sexto ano da rede municipal de Caxias do Sul, no Rio Grande do Sul. Revista Paulista de Pediatria, 31(1), 65-70. https://doi.org/10.1590/S010305822013000100011.

Lemes, D. C. M., Câmara, S. G., Alves, G. G., \& Aerts, D. (2018). Satisfação com a imagem corporal e bem-estar subjetivo entre adolescentes escolares do ensino fundamental da rede pública estadual de Canoas/RS, Brasil. Ciência \& Saúde Coletiva, 23, 4289-4298. https://doi.org/10.1590/1413812320182312.14742016.

Malta, D. C., Mascarenhas, M. D. M., Porto, D. L., Duarte, E. A., Sardinha, L. M., Barreto, S. M., \& Morais Neto, O. L. (2011). Prevalência do consumo de álcool e drogas entre adolescentes: análise dos dados da Pesquisa Nacional de Saúde Escolar. Revista Brasileira de Epidemiologia, 14, 136146. https://doi.org/10.1590/S1415-790X2011000500014.

Oliveira, M. M., Campos, M. O., Andreazzi, M. A. R., \& Malta, D. C. (2017). Características da pesquisa nacional de saúde do escolar-PeNSE. Epidemiologia e Serviços de Saúde, 26, 605-616. https://doi.org/10.5123/S1679-49742017000300017.

OMS. Actas oficiales de la OMS. (2001). n. 2, p.100.

Paz, F. M., Teixeira, V. A., Pinto, R. O., Andersen, C. S., Fontoura, L. P., Castro, L. C., Pattussi, M. P., \& Horta, R. L. (2018). Promoção de saúde escolar e uso de drogas em escolares no Sul do Brasil. Revista de Saúde Pública, 52(58), 1-11. https://doi.org/10.11606/s1518-8787.2018052000311.

Pinheiro, A. R. O., Freitas, S. F. T., \& Corso, A. C. T. (2004). 10.1590/s1415-52732004000400012. Revista de Nutrição, 17(4), 523-533. https://doi.org/10.1590/s1415-52732004000400012.

Silva, A. S. N., Silva, B. L. C. N., Silva Júnior, A. F., Silva, M. C. F., Guerreiro, J. F., \& Araújo, A. S. C. (2015). Início da vida sexual em adolescentes escolares: um estudo transversal sobre comportamento sexual de risco em Abaetetuba, Estado do Pará, Brasil. Revista Pan-Amazônica de Saúde, 6(3), 8. https://doi.org/10.5123/s2176-62232015000300004.

Silva, A. T., Jacob, M. H. V. M., \& Hirdes, A. (2015). Conhecimento de adolescentes do ensino médio sobre DST/AIDS no sul do Brasil. Aletheia, 46, 34-49. 
Silva, G. S., Lourdes, L. A., Barroso, K. de A., \& Guedes, H. M. (2015). Comportamento sexual de adolescentes escolares. Revista Mineira de Enfermagem, 19(1), 154-166. https://doi.org/10.5935/1415-2762.20150013.

Silva, L. P. R., Tucan, A. R. O., Rodrigues, E. L., Del Ré, P. V., Sanches, P. M. A., \& Bresan, D. (2019). Insatisfação da imagem corporal e fatores associados: um estudo em jovens estudantes universitários. Einstein, 17(4), eAO4642--eAO4642. https://doi.org/10.31744/einstein_journal/2019ao4642.

Silva, R. A. R., Nelson, A. R. C., Duarte, F. H. S., Prado, N. C. C., Holanda, J. R. R., \& Costa, D. A. R. S. (2016). Adolescent students knowledge about transmition, prevention and risky behavior related to STD/HIV/AIDS. Revista de Pesquisa: Cuidado é Fundamental Online, 8(4), 5054-5061.

Souza, E. A., Barbosa Filho, V. C., Nogueira, J. A. D., \& Azevedo Júnior, M. R. (2011). Atividade física e alimentação saudável em escolares brasileiros: revisão de programas de intervenção. Cadernos de Saúde Pública, 27(8), 1459-1471. https://doi.org/10.1590/S0102-311X2011000800002.

WHO. (2020). World Health Organization. 2020. What is a health promoting school?. https://www.who.int/school_youth_health/gshi/hps/en/. Acesso Em: 07 de outubro de 2020.

Histórico do artigo:

Recebido: 25 de novembro de 2020 .

Aprovado: 15 de janeiro de 2021.

Disponível online: 14 de abril de 2021.
Licenciamento: Este artigo é publicado na modalidade Acesso Aberto sob a licença Creative Commons Atribuição 4.0 (CC-BY 4.0), a qual permite uso irrestrito, distribuição, reprodução em qualquer meio, desde que o autor e a fonte sejam devidamente creditados. 